\title{
RATIONAL POINTS IN HENSELIAN DISCRETE VALUATION RINGS
}

BY MARVIN J. GREENBERG

Communicated by P. Cohen, February 25, 1966

Let $R$ be a Henselian discrete valuation ring (i.e., one in which Hensel's Lemma holds; examples are complete discrete valuation rings, the ring of algebraic $p$-adic integers, the ring of convergent power series in one variable over a complete valued field [1]). Let $t$ be a generator of the maximal ideal, $K$ the field of fractions, $R^{*}$ the completion of $R$; let $K^{*}$ be the field of fractions of $R^{*}$. If $F$ $=\left(F_{1}, \ldots, F_{r}\right)$ is a system of $r$ polynomials in $n$ variables with coefficients in $R$, let $F R[X]$ be the ideal in $R[X]$ generated by $F_{1}, \cdots, F_{r}$. If $x$ is an $n$-tuple with coordinates in $R$, set $F(x)=\left(F_{1}(x)\right.$, $\left.\cdots, F_{r}(x)\right)$ :

Theorem 1. Assume that $K^{*}$ is separable over $K$. Then there are integers $N \geqq 1, c \geqq 1$ depending on $F R[X]$ such that for any $\nu \geqq N$ and any $x$ in $R$ such that

$$
F(x) \equiv 0\left(\bmod t^{\nu}\right)
$$

there exists $y$ in $R$ such that

$$
\begin{aligned}
y & \equiv x\left(\bmod t^{[v / c]}\right), \\
F(y) & =0 .
\end{aligned}
$$

Corollary 1. Let $Y$ be a prescheme of finite type over $R$. Then there are integers $N \geqq 1, c \geqq 1$ depending on $Y$ such that for $\nu \geqq N$ and for any point $x$ of $Y$ in $R / t^{\nu}$, the image of $x \bmod t^{[\nu / c]}$ lifts to a point of $Y$ in $R$.

Corollary 2. $Y$ has a point in $R$ if and only if $Y$ has a point in $R / t^{\nu}$ for all $\nu$.

Corollary 1 follows from Theorem 1 by taking a finite covering of $Y$ by affine opens $Y_{i}$ and remarking that $Y(S)=\cup_{i} Y_{i}(S)$ for any local $R$-algebra $S$.

Let $Y=\operatorname{Spec} R[X] / F R[X]$ be the affine scheme over $R$ defined by $F, Y_{K}$ the scheme over $K$ obtained by base change. In the special case that $R$ is complete and $Y_{K}$ is irreducible and smooth over $K$, Néron [2; Proposition 20, p. 38] has proved a different form of Theorem 1.

The proof of Theorem 1 goes by Noetherian induction on $Y_{K}$. One reduces easily to the case $Y$ reduced and irreducible. Then there are two cases, depending on whether the function field of $Y_{K}$ is separable 
or not over $K$. In the separable case, the key is Newton's Lemma, which enables us to refine $x$ to a zero provided that $Y$ is a complete intersection and the Jacobian matrix of $F$ at $x$ has the maximal rank $\bmod t^{[(\nu-1) / 2]}$; if the latter condition fails, then the inductive hypothesis enables us to refine $x$ to a zero on the singular locus of $Y_{K}$. In the inseparable case, there is a finite purely inseparable extension $K^{\prime}$ of $K$ such that $Y_{K^{\prime}}$ is not reduced. Since $K^{*}$ is separable over $K$, the integral closure $R^{\prime}$ of $R$ in $K^{\prime}$ is a finite $R$-module [ $3 ; 0_{\mathrm{IV}}, 23.1 .7$ (ii)]. Then techniques of [4] enable us to pull $\left(Y_{R^{\prime}}\right)_{\text {red }}$ down to a proper closed subscheme of $Y$ for which the inductive hypothesis applies.

The detailed proof will appear in Publ. Math. Inst. Hautes Études.

As one application of Theorem 1, recall that a domain $R$ is called $C_{i}$ if any form with coefficients in $R$ of degree $d$ in $n$ variables with $n>d^{i}$ has a nontrivial zero in $R$.

Theorem 2. If $k$ is $a C_{i}$ field, then the field $k((t))$ of formal power series in one variable tover $k$ is $C_{i+1}$.

This generalizes some results of Lang [5], who did the cases $i=0$ and $k$ finite.

It suffices to prove that $R=k[[t]]$ is $C_{i+1}$. By Lang [5], $k[t]$ is $C_{i+1}$. Hence the hypersurface $H$ in projective $(n-1)$-space defined by the given form has a point in the ring $R / t^{\nu}$ for all $\nu$. By Corollary 2, $H$ has a point in $R$.

Note. The same type of argument yields a short proof of Lang's theorem that if $R$ is a Henselian discrete valuation ring with algebraically closed residue field, such that $K^{*}$ is separable over $K$, then $R$ is $C_{1}$. For by Corollary 2 , we may assume $R$ complete, and since $C_{1}$ is inherited by finite extensions, we may also assume $R$ unramified. Then the argument given in $[5 ;$ p. 384] shows that $H$ has a point in $R / t^{\nu}$ for all $\nu$.

Acknowledgment. Communications with M. Artin, P. Cohen, and M. Raynaud have been very helpful.

\section{REFERENCES}

1. M. Nagata, Local rings, Interscience, New York, 1962.

2. A. Néron, Modeles minimaux des variêtés abéliennes sur les corps locaux et globaux, Inst. Hautes Études Sci. Publ. Math. No. 21, Paris, 1964.

3. A. Grothendieck and J. Dieudonné, Éléments de géométrie algébrique. IV (première partie), Inst. Hautes Êtudes Sci. Publ. Math. No. 20, Paris, 1964.

4. M. Greenberg, Schemata over local rings, Ann. of Math. 73 (1961), 624-648

5. S. Lang, On quasi-algebraic closure, Ann. of Math. 55 (1952), 373-390.

NORTHEASTERN UNIVERSITY 